\title{
Solid haemangioblastomas of the posterior fossa: radiological features and results of surgery
}

\author{
STEVEN YOUNG, ALAN E RICHARDSON \\ From the Department of Neurosurgery, Atkinson Morley's Hospital, Wimbledon, UK
}

SUMMARY Cerebellar haemangioblastomas are benign neoplasms which have been reported to have a favourable prognosis. The authors report the experience of Atkinson Morley's Hospital, London with 14 cases of solid haemangioblastoma since the introduction of the CT scan. Death or a poor result occurred in $50 \%$ of cases. Incomplete excision carried a high risk of complications. The CT scan appearances were too non specific to make an accurate preoperative diagnosis, the lesions usually being mistaken for meningiomas. Vertebral angiography can be of considerable help in identifying their vascular anatomy.

Cerebellar haemangioblastomas are benign neoplasms which comprise $7 \cdot 3$ to $12 \%$ of all posterior fossa tumours. ${ }^{1-4}$ The basic surgical approach to them has remained unchanged since first described by Cushing and Bailey, and by Dandy in $1928 .^{56}$ The majority of haemangioblastomas are cystic which, if completely excised, have a favourable outcome after surgery. To date, there has been little detail in the literature about the less common solid variety of haemangioblastoma and the particular problems they present, both with respect to diagnosis and surgical removal. Previous series have all grouped cystic and solid lesions together, and were collected before the introduction of computed tomography.

The experience of Atkinson Morley's Hospital, London, with solid haemangioblastomas, since the inception of the CT scan, has been reviewed, and the cases analysed with particular respect to radiological appearances and operative findings. The results of surgery are discussed.

\section{Summary of cases}

Between 1973 and 1984, 52 patients underwent suboccipital craniectomy for a posterior fossa tumour that was later diagnosed histologically as a haemangioblastoma. Fourteen of these were categorised as solid haemangioblastomas. Within this group, there were eight males and six females with an average age of 43.4 (range 20-73) years. The presenting symptoms and signs were no different from those described in a previous series reported from this hospital. ${ }^{7}$ The average

Address for reprint requests: Mr S Young, Department of Neurosurgery, Atkinson Morley's Hospital, London SW20 0NE, UK.

Received 30 May 1986. Accepted 23 June 1986 duration of symptoms prior to presentation was 7 months, the commonest being headache, nausea and loss of balance. Papilloedema and gait ataxia were the commonest findings ( $85 \%$ and $65 \%$ respectively). One patient in the group underwent his second operation for a recurrent haemangioblastoma.

The pathological features of the lesions were relatively uniform. Macroscopically, they appeared as reddish brown, often multilobulated nodules. The histological appearance was that of a network of sinusoidal vessels, usually with areas of patchy thrombosis and necrosis, and an intervening reticular stroma.

\section{Radiological diagnosis}

Twelve patients had skull radiographs which were non contributory. Two patients, in the early days of CT scanning, had also undergone ventriculography which showed marked dilatation of the third and lateral ventricles with displacement of the aqueduct and fourth ventricle from the midline.

All 14 patients had full CT scans, and all of these were available for review. The results are summarised in table 1.

Table 1 Summary of CT findings in 14 patients with solid haemangioblastomas of the posterior fossa

\begin{tabular}{|c|c|}
\hline$C T$ characteristics & Number of patients \\
\hline \multicolumn{2}{|l|}{ Density } \\
\hline low & 12 \\
\hline mixed & 2 \\
\hline isodense & 1 \\
\hline \multicolumn{2}{|l|}{ Contrast Enhancement } \\
\hline prominent & 11 \\
\hline moderate & 2 \\
\hline ring enhancement & 1 \\
\hline \multicolumn{2}{|l|}{ Location } \\
\hline cerebellar hemisphere & $10(6 \mathrm{~L} ; 4 \mathrm{R})$ \\
\hline $\begin{array}{l}\text { C-P angle } \\
\text { midline }\end{array}$ & 2 \\
\hline Hydrocephalus & 14 \\
\hline
\end{tabular}





Fig 1 CT scan showing appearance of solid haemangioblastoma (a) before and (b) after enhancement with contrast medium.

The tumours were generally of low density, arising in one or other cerebellar hemisphere, with distortion and displacement of the fourth ventricle. Hydrocephalus was seen in every case. Very prominent, almost homogeneous enhancement with contrast medium was a feature common to 12 cases (fig 1). Both cerebellopontine (CP) angle haemangioblastomas were seen on uncontrasted CT scans as a zone of reduced soft tissue density, within which there were tiny areas of high attenuation attributed to calcification. After injection of contrast medium, strong enhancement was seen. One of the lesions in the midline was isodense, and showed ring enhancement with contrast. None of the CT scans showed recent evidence of haemorrhage.

While the haemangioblastomas in the midline and $\mathrm{CP}$ angle might be expected to cause difficulties with diagnosis, it is of interest that in 10 patients, the primary radiological diagnosis on the basis of CT scan, was meningioma. The possibility of haemangioblastoma was raised in three cases, but stated to be the most likely diagnosis in only one.

Vertebral angiography was requested in three patients. Two of the angiograms were described as being "diagnostic" of haemangioblastoma, showing a clear vascular blush with feeding vessels (fig 2). In one case these were clearly identified as both posterior inferior cerebellar (PICA) arteries, and one superior cerebellar (SCA) artery. One angiogram failed to demonstrate any abnormality, yet on CT scan, and at surgery, a moderate sized nodule was found within a cerebellar hemisphere.

\section{Operative findings}

All 14 patients underwent suboccipital craniectomy, 10 in the sitting and four in the "park bench" positions. Debate concerning the relative merits of the sitting and prone or semi-prone positions is likely to prevail for some time. There were no recorded cases of air embolism in our series, and the potential problems of congestion and poor operative conditions were not encountered in the four patients who were positioned semi-prone (park bench). The operative findings are summarised in table 2 .

There appeared to be two distinct types of lesion. The first, a relatively well circumscribed nodule entirely within cerebellar tissue, with a well defined plane between it and

Table 2 Summary of operative findings in 14 cases of solid haemangioblastomas of the posterior fossa

\begin{tabular}{ll}
\hline Operative findings & No of patients \\
\hline Tonsillar herniation & 7 \\
Nodular lesions, confined to & 6 \\
cerebellar hemisphere & 2 \\
Nodular lesions within the vermis & 2 \\
Complex lesions, extending to: & 2 \\
tentorium & 1 \\
C-P angle & 2 \\
Floor of post. fossa & 9 \\
Brainstem & \\
\hline
\end{tabular}



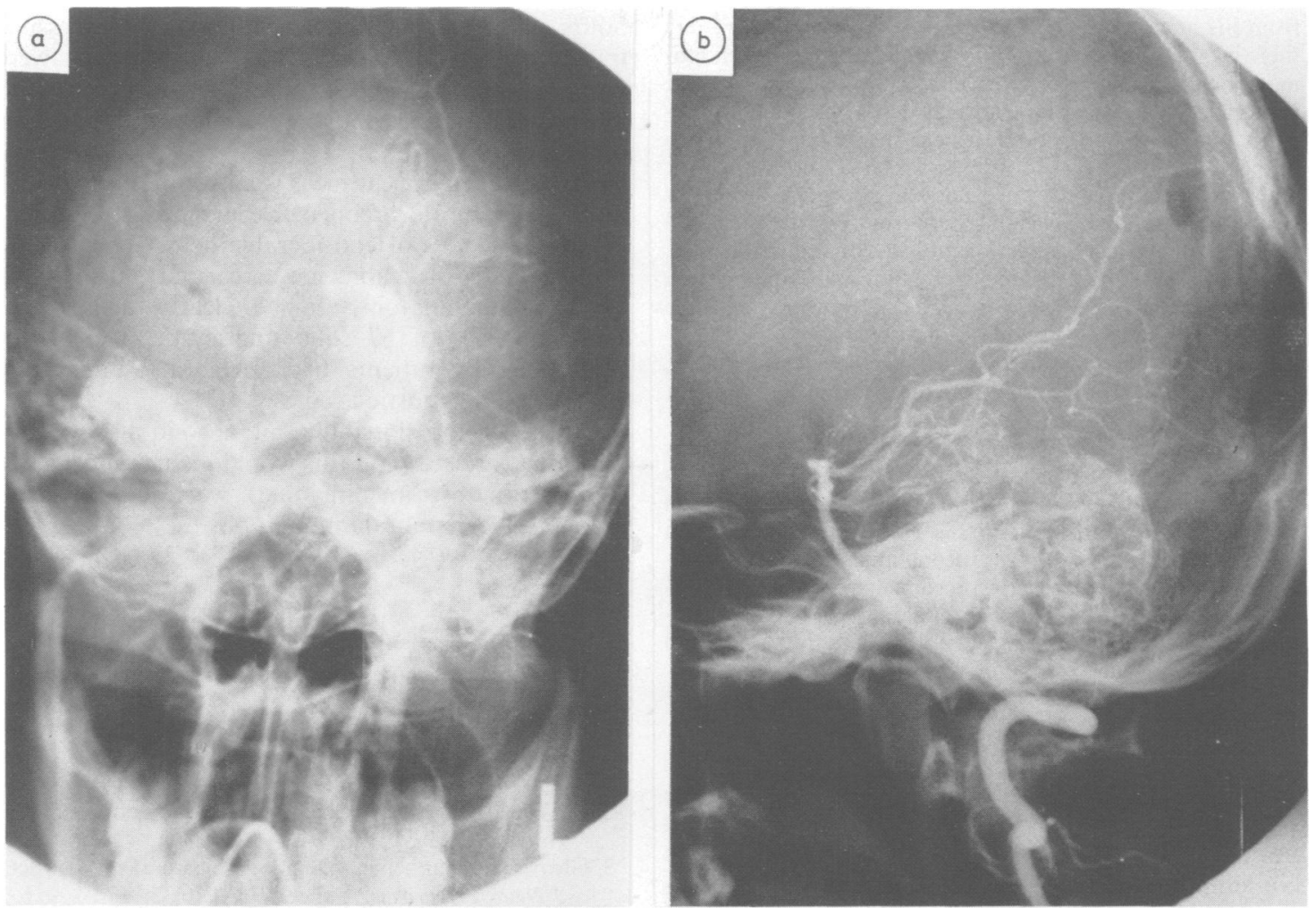

Fig 2 Vertebro-basilar angiogram showing typical solid haemangioblastoma. (a) anteroposterior and (b) lateral view.

normal brain. The second, or diffuse, consists of a complex vascular network with feeding arteries and arterialised veins, arising a few millimetres below the suface of the cerebellum, extending to the surface and beyond to the floor of the posterior fossa, inferior aspect of the tentorium, brain stem or $\mathrm{CP}$ angle.

The main intraoperative problem with both types of lesion was haemorrhage. In the case of the nodular type, this was caused in some cases by attempts at biopsy, the diagnosis not having been considered beforehand. The diffuse type of lesion, predictably, was more difficult to excise, but in some cases there was a covering of pia delineating a potential plane of dissection, through which feeding vessels could be systematically clipped or coagulated. Eventually, the surgeon was left with a vascular pedicle which could be clipped and transected.

Resection was incomplete in five patients. In four cases, troublesome intraoperative bleeding precluded total excision, blood loss being in excess of 1.5 litres in two patients despite induction of hypotension to a systolic pressure of $70 \mathrm{~mm} \mathrm{Hg}$ during the procedure. In one patient complete removal was impossible because the haemangioblastoma extended from the floor of the posterior fossa around the brain stem.

\section{Outcome}

A summary of the results of surgery is given in table 3 . There were five early postoperative deaths (that is, within 28 days). In three cases, excision had been complete, and death was attributed by CT scan, or by necropsy, to a postoperative haematoma. In two such cases, clinical deterioration was evident within hours of surgery, and speedy evacuation of the clots made no difference either to the immediate levels of consciousness or the outcome. Another patient died 27 days after surgery with CT evidence of bleeding from a partially excised CP angle haemangioblastoma.

One patient, with a vermian lesion, was well until the fourth postoperative day when she died suddenly with no warning of neurological or other major systemic deterioration. Necropsy revealed that there had been an enormous fresh haemorrhage into the cerebellum, but there was no evidence of residual tumour. The last patient had his haemangioblastoma excised totally, recovered uneventfully

Table 3 Results of surgery in 14 patients with solid haemangioblastomas and a comparison with 38 cases of cystic haemangioblastoma, at Atkinson Morley's Hospital from 1973-1984

\begin{tabular}{lllcc}
\hline Type of lesion & Deaths & Poor & Good & Excellent \\
\hline Solid (14) & 5 & 2 & 3 & 4 \\
Cystic (38) & 2 & 2 & 12 & 22 \\
\hline
\end{tabular}


from his anaesthetic, but on the second postoperative day there was a gradual deterioration in his conscious level, ultimately with loss of respiratory effort and limb responses. A postoperative CT scan was normal. Unfortunately, no necropsy was carried out, and death was attributed clinically to brain stem oedema.

Of the two patients who made a poor recovery after surgery, one suffered a haemorrhage from a partially excised lesion two days after operation which required evacuation. The other developed chronic depression and required long term psychiatric care. He was unable to return to full time work despite making a good physical recovery.

Transient worsening of ataxia and diplopia was common, but seven patients eventually made a good or excellent recovery. One remarkable patient had a midline recurrence removed 5 years following initial surgery, followed two years later by excision of what was described as a frontal intraventricular haemangioblastoma. He subsequently presented with further deterioration in his gait, and a myelogram revealed multiple seedlings throughout the spinal subarachnoid space which were presumed to represent metastatic haemangioblastoma. This could not be verified histologically, but the patient improved with radiotherapy and has had a long survival.

\section{Discussion}

The gratifying response to treatment of haemangioblastomas, claimed by Mondkar et al, ${ }^{7}$ clearly does not apply to the solid group of lesions. Even with the contemporary advantages of the operating microscope, bipolar diathermy and intraoperative hypotension, death or a poor result occurred in half of the 14 patients in this series. Five of the seven adverse results were related to postoperative haemorrhage. This correlates well with Jeffrey's subgroup of 20 solid haemangioblastomas where the mortality approached $50 \%$ (five immediate deaths and four from recurrences). It is difficult to know how these results can be improved. In all the patients in our series haemostasis was deemed satisfactory, and the blood pressure restored to normal levels before wound closure. Blood loss was replaced within 12 hours of surgery, and when it had been excessive, fresh frozen plasma and platelet concentrates were administered. Blood pressure was carefully controlled in the first 24 hours following surgery to avoid surges of hypertension.

The CT scan appearances were too non specific to make an accurate preoperative diagnosis. Vertebral angiography was of considerable help in two of the three patients on whom it was carried out allowing the vascular anatomy to be clearly defined. In Jeffrey's series of 67 solid and cystic haemangioblastomas, 14 patients had vertebral angiography with $100 \%$ diagnostic accuracy.

Whenever the possibility of a solid haemangioblastoma is raised (and this would include most cases of posterior fossa meningioma), we would advocate preoperative vertebral angiography.

\section{References}

1 Jeffreys R. Clinical and surgical aspects of posterior fossa haemangioblastomas. $J$ Neurol Neurosurg Psychiatry 1975;38:105-11.

2 Olivecrona $\mathrm{H}$. The cerebellar angioreticulomas. $J \mathrm{Neu}$ rosurg 1952;9:317-30.

3 Gleave J. Cerebellar haemangioblastomas (abstract). J Neurol Neurosurg Psychiatry 1970;33:717.

4 Dastur DK, Lalitha VS. Pathological analysis of intracranial space occupying lesions in 1,000 cases including children. Part 3, Vascular tumours and hamartomas; meningiomas, schwannomas. $J$ Neurol Sci 1970;11:501-35.

5 Cushing H, Bailey P. Tumours Arising from the Blood Vessels of the Brain. Springfield: Thomas, 1928.

6 Dandy WE. Venous abnormalities and angiomas of the brain. Arch Surg 1928;17:715-93.

7 Mondkar VP, McKissock W, Russell EWR. Cerebellar haemangioblastomas. Br J Surg 1967;54:45-59.

8 Singounas EG. Haemangioblastomas of the central nervous system. Acta Neurochirurg 1978;44:107-13. 